\title{
Ewing's Sarcoma of Kidney- a Rare Case
}

\author{
Bikash Bikram Thapal, Narayan Thapa ${ }^{1}$, Bharat Bahadur Bhandari ${ }^{1}$, Bhairab Kumar Hamal \\ ${ }^{1}$ Department of Surgery, Shree Birendra Hospital, Kathmandu, Nepal.
}

\begin{abstract}
Ewing's sarcoma of kidney is rare and aggressive tumor of young adult with diagnostic challenges. Clinical presentations and imaging are non specific. The diagnosis depends on histopathology and immunohistochemistry and treatment encompass multimodality approach. We present here a case of Ewing's sarcoma kidney of a young adult managed in our institution.
\end{abstract}

Keywords: ewing's sarcoma; nephrectomy; immunohistochemistry; CD99; therapy.

\section{Introduction}

Peripheral primitive neuroectodermal tumor/Ewing's sarcoma (pPNET/EWS) is an aggressive type of sarcoma predominantly a malignant bone or soft tissue tumor of children and young adults. Primary Ewing's sarcoma of the kidney is very rare tumor that was first described in 1975 by Seemayer and colleagues ${ }^{1}$. The current study presents the first known case of renal pPNET in our institution. Renal ES usually affects at the age between 20 and 30 years. $25 \%-50 \%$ present with metastatic disease commonly in the lung, liver, and bone ${ }^{2}$. Tumor thrombi are reported frequently ${ }^{3,4}$. Treatment strategies for renal ES include surgery, chemotherapy, and radiation ${ }^{2}$.The main challenge of ES remains proper diagnosis and its confirmation facilitating a patient's adequate treatment in duetime ${ }^{5}$.

\section{Case Report}

A 23 year serviceman was evaluated for right flank pain and ultrasonography showed right renal mass. Urine routine examination, complete blood count, renal function, chest x-ray were within normal limits. Patient was subjected to contrast enhanced CT abdomen and pelvis (Figure 1) that revealed $8.4 \times 7.4$ $x 7.5 \mathrm{~cm}$ sized heterogeneously enhancing soft tissue density mass in mid pole of right kidney abutting liver, IVC and duodenum with tumor thrombus in right renal vein with radiological diagnosis of right renal cell carcinoma.
Patient underwent right radical nephrectomy from right subcostal approach. Grossly the kidney measured $14 \mathrm{x}$ $9 \times 9 \mathrm{~cm}$ and on cut section the nodular solid tumor measures $6 \times 5.4 \times 3 \mathrm{~cm}$ with areas of haemorrhage and necrosis (Figure 2). The postoperative period was uneventful.

Histopathological report was diffusely infiltrated small blue round cell population in sheets of varying size. PAS stain shows glycogen in many of the cells. An immunohistochemical panel demonstrated strong expression of CD99. S-100, CD-45, CD56, TDT,WT1, ketatins and desmin are negative. The final report was renal mass consistent with Ewing's Sarcoma (PNET). Patient is currently under combination chemotherapy ( EFT 2001 protocol)

\section{Discussion}

Stout described Ewing's sarcoma in 1918 in ulnar nerve that contain small round blue cells arranging into rosettes. John Ewing further characterized these tumors describing them in the diaphysis of long bones. These tumors are neural crest cells in origin belonging to a family of tumors called primitive neuroectoderm tumors (PNETs). pPNETs are part of the EWS family of tumors with EWS differing on the fact that it lacks neuroectodermal features . ${ }^{1,6}$

A peripheral primitive neueoectoderm tumor (pPNET) is a small round cell tumor that predominantly occurs in

\section{Correspondence:}

Bikash Bikram Thapa,

Department of Surgery, Shree Birendra Hospital, Chhauni.

E-mail:bbthapa@gmail.com. 
bone and soft tissue; however, pPNET is occasionally reported in visceral organs, such as urogenital, intra abdominal and intra thoracic organs. pPNET of the kidney is an aggressive disease with a high metastatic potential ${ }^{7}$.

Clinical symptoms and signs are non specific, comprising a triad of flank or abdominal pain, palpable mass, and hematuria in decreasing order. Systemic symptoms such as weight loss (14.5\%) and fever (9.7 $\%)$ are indicative of the underlying malignant disease. Laboratory findings are mostly unremarkable, but elevated lactate dehydrogenase (LDH) and (neuron specific enolase (NSE) levels were reported ${ }^{8}$. Apart from a poorly defined, infiltrative large mass with necrotic and hemorrhagic areas, generally imaging studies do not reveal characteristic signs.

Diagnosis of pPNET is based on morphologic, immunohistochemical and genetic analyses. Small, round cells with hyperchromatic nuclei, scant to moderate cytoplasms and occasional rosettelike structures are the hallmarks of pPNET of the kidney ${ }^{9}$. However, pPNET of the kidney must still be differentiated from other small round cell tumors, such as blastemalWilms' tumor, malignant lymphoma, small cell carcinoma, rhabdomyosarcoma, poorly differentiated synovial sarcoma and desmoplastic small roundcell tumors. Overexpression of surface membrane protein CD99 and nuclear staining of FLI1 (Friend Leukemia integration 1 transcription factor) are universal features of $\mathrm{pPNET}^{7,10}$.

Most cases of Ewing's sarcoma of kidney were diagnosed following nephrectomy. The main challenge of ES remains proper diagnosis and its confirmation facilitating a patient's adequate treatment in due time. ${ }^{5} \mathrm{ES}$ benefits from a combined treatment. ${ }^{11}$ The EURO-E.W.I.N.G. 99 study recommends a multiagent chemotherapy delivered prior (VIDE induction therapy) and continued after local control (consolidation therapy consisting of VAI or VAC) ${ }^{12}$.

Surgery is superior for local control and is frequently carried out as the first line of treatment. In addition, surgical intervention may encompass cavotomy in cases of venous tumor thrombus and metastasectomy, e.g. of pulmonary nodules. Irradiation of the surgical site with 50-60 Gys, results in complete responses in terms of reducing residual local disease however general consensus is still lacking ${ }^{13}$. Presence of tumor thrombus in the inferior vena cava is associated with a higher rate of pulmonary metastatic embolism so more intense chemotherapy and/or extended fields of radiation is warranted ${ }^{3,4,14,15}$. The survival expectancy of patients for localized disease is $70 \%$, whereas the outcome of metastatic disease at initial diagnosis remains poor, with survival rates from $9 \%$ to $41 \%{ }^{16}$. About $30 \%-40 \%$ of patients with ES experience tumor recurrence ${ }^{11}$.

\section{Conclusion}

ES is a rare kidney tumor that affects young adults with rapid clinical progression and significant mortality owing to late diagnosis, early metastasis, and advanced stage at presentation. Therefore, patients benefit from a correct and early diagnostic approach, followed by combined modality therapy.

\section{References}

1. Seemayer TA, Thelmo $W L$, Bolande $R P$, Wiglesworth FW. Peripheral neuroectodermal tumors. Perspect Pediatr Pathol. 1975;2:151-72.

2. Ellinger J, Bastian PJ, Hauser S, Biermann K, Muller SC. Primitive neuroectodermal tumor: rare, highly aggressive differential diagnosis in urologic malignancies. Urology. 2006 Aug;68(2):257-62.

3. Karpate A, Menon $S$, Basak R, Yuvaraja TB, Tongaonkar HB, Desai SB. Ewing sarcomal primitive neuroectodermal tumor of the kidney: clinicopathologic analysis of 34 cases. Ann Diagn Pathol. 2012 Aug;16(4):267-74.

4. Lazzara BM, Scalcione LR, Garnet DJ, Geller $M$, Katz DS. Radiology-pathology conference: primary perinephric and renal extraosseous Ewing's sarcoma. Clin Imaging. 2012 JanFeb;36(1):77-9.

5. Casella R, Moch H, Rochlitz C, Meier V, Seifert $B$, Mihatsch MJ, et al. Metastatic primitive neuroectodermal tumor of the kidney in adults. Eur Urol. 2001 May;39(5):613-7.

6. Ohgaki K, Horiuchi K, Mizutani S, Sato M, Kondo Y. Primary Ewing's sarcoma/primitive neuroectodermal tumor of the kidney that responded to low-dose chemotherapy with ifosfamide, etoposide, and doxorubicin. Int J Clin Oncol. 2010 Apr;15(2):210-4.

7. Risi E, Iacovelli R, Altavilla A, Alesini D, Palazzo $A$, Mosillo $C$, et al. Clinical and pathological features of primary neuroectodermal tumor/ Ewing sarcoma of the kidney. Urology. 2013 
Aug;82(2):382-6.

8. Bing Z, Zhang P, Tomaszewski JE, Maclennan GT. Primary Ewing sarcoma/primitive neuroectodermal tumor of the kidney. $J$ Urol. 2009 Mar;181(3):1341-2.

9. Parham DM, Roloson GJ, Feely M, Green DM, Bridge JA, Beckwith JB. Primary malignant neuroepithelial tumors of the kidney: a clinicopathologic analysis of 146 adult and pediatric cases from the National Wilms' Tumor Study Group Pathology Center. Am J Surg Pathol. 2001 Feb;25(2):133-46.

10. Saxena R, Sait S, Mhawech-Fauceglia P. Ewing sarcoma/primitive neuroectodermal tumor of the kidney: a case report. Diagnosed by immunohistochemistry and molecular analysis. Ann Diagn Pathol. 2006 Dec;10(6):363-6.

11. Miser JS, Kinsella TJ, Triche TJ, Steis R, Tsokos $M$, Wesley $R$, et al. Treatment of peripheral neuroepithelioma in children and young adults. $J$ Clin Oncol. 1987 Nov;5(11):1752-8.
12. Zollner S, Dirksen U, Jurgens H, Ranft A. Renal Ewing tumors. Ann Oncol. 2013 Sep;24(9):245561.

13. Thomas JC, SebekBA, Krishnamurthi V. Primitive neuroectodermal tumor of the kidney with inferior vena cava and atrial tumor thrombus. $J$ Urol. 2002 Oct;168(4 Pt 1):1486-7.

14. Castro EC, Parwani AV. Ewing sarcomal primitive neuroectodermal tumor of the kidney: two unusual presentations of a rare tumor. Case Rep Med. 2012;2012:190581.

15. Mohsin R, Hashmi A, Mubarak M, Sultan $G$, Shehzad A, Qayum $A$, et al. Primitive neuroectodermal tumor/Ewing's sarcoma in adult uro-oncology: A case series from a developing country. Urol Ann. 2011 May;3(2):103-7.

16. Ladenstein $R$, Potschger $U$, Le Deley $M C$, Whelan J, Paulussen $M$, Oberlin $O$, et al. Primary disseminated multifocal Ewing sarcoma: results of the Euro-EWING 99 trial. J Clin Oncol. 2010 Jul 10;28(20):3284-91.

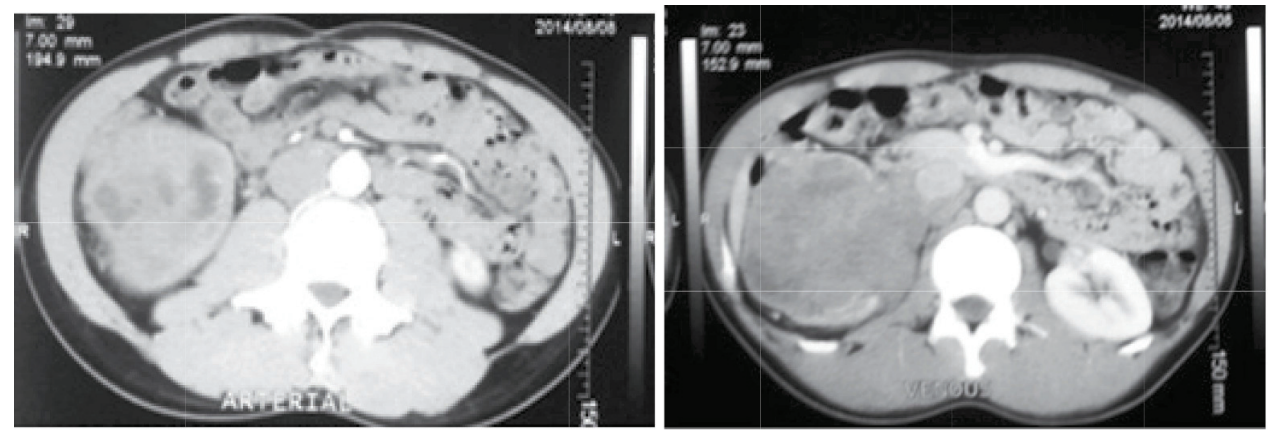

Fig.1

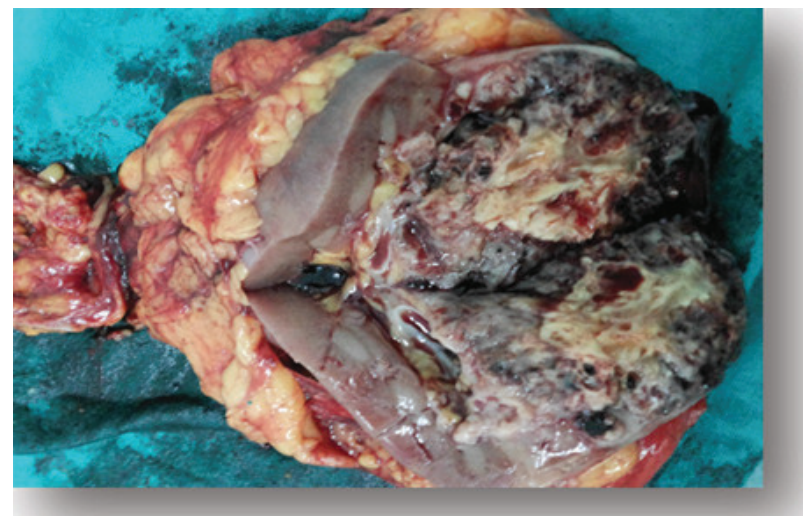

Figure 2 